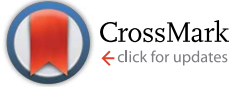

Cite this: RSC Adv., 2017, 7, 3025

Received 20th October 2016 Accepted 5th December 2016

DOI: 10.1039/c6ra25505a

www.rsc.org/advances

\section{Superlubricity of hydrogenated carbon films in a nitrogen gas environment: adsorption and electronic interactions at the sliding interface}

\begin{abstract}
Chengbing Wang, ${ }^{\text {ab }}$ Bingrui $\mathrm{Li}^{\mathrm{c}}{ }^{\mathrm{X}}$ Xiaoming Ling ${ }^{\mathrm{d}}$ and Junyan Zhang ${ }^{\text {*b }}$
The tribological behavior of hydrogenated amorphous carbon film (a-C:H) is known to be very sensitive to the external environment. In an $\mathrm{N}_{2}$ environment, a-C:H can maintain long-term superlubricity. Currently, no convincing explanation is available for the mechanism through which $\mathrm{N}_{2}$ significant decreases the friction coefficients. In this work, we explain the tribological behavior of a-C:H films by accounting for the internal electronic structure of ambient gas molecules, their atomic electronegativity, the surface bonding state of $\mathrm{a}-\mathrm{C}: \mathrm{H}$, and the synergistic effects of these factors. Here, we proposed a mechanism based on the unique electronic structure of $\mathrm{N}_{2}$ molecules. $\mathrm{N}_{2}$ has two lone electron pairs, and its lone-pair electrons interact with surface $\mathrm{C}-\mathrm{H}$ bonds of $\mathrm{a}-\mathrm{C}: \mathrm{H}$, forming $-\mathrm{C}-\mathrm{H} \cdots: \mathrm{N} \equiv \mathrm{N}$ : interactions that are similar to the hydrogen bonds in water. This causes $\mathrm{N}_{2}$ to be adsorbed on the film's surface. As two surfaces approach each other, the other lone-pair electrons in $\mathrm{N}_{2}$ become close, generating electrostatic repulsion and resulting in super-low friction. Based on the above analysis, we calculated the friction forces of a self-mated a$\mathrm{C}: \mathrm{H}$ film in different environments (vacuum, $\mathrm{N}_{2}, \mathrm{O}_{2}$ ) using density functional theory. These theoretical calculations matched experimental results, indicating that the proposed approach is reasonable.
\end{abstract}

\section{Introduction}

Because of their unique physical, mechanical, and tribological properties, carbon-based films have been used as solid lubricants in a broad range of applications from microelectromechanical systems (MEMS) to macro-scale mechanical components, and they have attracted much attention from academic and industrial researchers. ${ }^{1-3}$ The design of carbonbased films with superlubricity and the study of their tribological mechanisms have been popular research topics. ${ }^{4,5}$ The different preparation methods and deposition conditions used to form amorphous carbon films lead to different atomic bond configurations (e.g., $\mathrm{C}-\mathrm{H}, \mathrm{C}-\mathrm{C}, \mathrm{sp}^{2}$, or $\mathrm{sp}^{3}$ ) and different bond proportions in these films, which further determine the differences in their physical and chemical properties. ${ }^{6}$ Depending on its hydrogen content, amorphous carbon film can be divided into two main categories as either hydrogen-free diamond-like carbon (DLC) film (e.g., ta-C and a-C) or hydrogenated DLC film (e.g., a-C:H and ta-C:H). ${ }^{3,6}$ Because of the structural

${ }^{a}$ School of Materials Science \& Engineering, Shaanxi University of Science and Technology,Xi'an, Shaanxi 710021, China. E-mail: wangchengbing@gmail.com; zhangjunyan@licp.cas.cn

${ }^{b}$ State Key Laboratory of Solid Lubrication, Lanzhou Institute of Chemical Physics, Chinese Academy of Science, Lanzhou 730000, China

${ }^{c}$ College of Chemistry and Chemical Engineering, Lanzhou University, Lanzhou 730000, China

${ }^{d}$ School of Mechatronic Engineering, Lanzhou Jiaotong University, Lanzhou 730070, China diversity of carbon-based films and the sensitivity of their tribological behavior to test conditions (e.g., environmental atmosphere), the current understanding of their friction mechanism is limited, restricting their use in practical applications. ${ }^{5,7}$ In order to determine the tribological behavior of amorphous carbon films, researchers have examined the effects of friction conditions (e.g., environmental atmosphere, friction pair materials, applied load, and sliding speed) on the tribological and wear behaviors of amorphous carbon films., ${ }^{3,8-17}$ Scholars have proposed some lubrication mechanisms for carbon-based films that are based on different phenomena, including friction-induced graphitization mechanism, ${ }^{\mathbf{1 8 - 2 0}}$ localized shear mechanism, ${ }^{21-23}$ and passivation mechanism (saturation of dangling carbon bonds at the sliding interface with passivating species). ${ }^{24-29}$

In the graphitization mechanism, a "graphitization" transfer layer forms at the sliding interface. In this theory, the graphitization transition that is induced by an increase in friction temperature determines the film's friction, and the formation of a transfer layer with a low shear stress is the primary cause of low-friction behavior in carbon films. ${ }^{18-20}$ In fact, the intrinsic chemical composition of the transfer layer determines the carbon film's tribological behavior rather than the graphitization characteristics of the transfer layer. ${ }^{\mathbf{1 5 , 3 0}}$ Recent studies have challenged this traditional mechanism in which graphitization leads to low friction. ${ }^{\mathbf{1 4 , 3 1 , 3 2}}$ In the localized shear mechanism, ultra-low friction is achieved when there is a strong phase transition in a very thin local regions at the sliding interface and 
a high localized shear stress. ${ }^{21-23}$ However, during contact and shear stress, the carbon phase transition inevitably occurs at the sliding interface. Since $\mathrm{sp}^{2}$ carbon is relatively soft and has a low shear strength, rehybridization occurs at the sliding interface during high-stress friction processes through the complicated atomic interactions and rearrangements that accompany bond breaking. ${ }^{27,33}$ Currently, the surface passivation mechanism is the most widely accepted.

For hydrogen-free DLC film with a relatively simple chemical composition has simpler tribological behavior that involves a less controversial mechanism. In these systems, tribological behavior is mainly determined by dangling surface bonds. In vacuum or an inert atmosphere, the dangling surface bonds of hydrogen-free DLC film act as a strong adhesive at the friction interface, leading to very high coefficient of friction (COF) of up to 0.4 or more. ${ }^{\mathbf{1 2 , 2 6}}$ In high humidity environments, water and $\mathrm{O}_{2}$ molecules effectively eliminate dangling surface bonds, reducing the $\mathrm{COF}^{27}$

However, the structures of hydrogenated DLC films are more complicated and exhibit more unique tribological behaviors due to the addition of hydrogen. The friction behaviors of a-C:H films are highly atmosphere dependent. Overall, the tribological behaviors of a-C:H films are determined by the synergistic effects of the film's internal structures and the external factors related to the tribological test conditions. The film's hydrogen content plays a decisive role in determining its tribological behavior, and films with higher hydrogen contents usually exhibit superior tribological properties. ${ }^{\mathbf{8} 34}$ In dry $\mathrm{N}_{2}$ gas atmosphere, a-C:H film can more easily achieve a low COF even superlubricity. ${ }^{\mathbf{1 4 , 3 3 , 3 5 , 3 6}}$ For example, the a-C:H film prepared by Argone National Laboratory has an extremely low COF (0.003) and a very long wear life, exhibiting no failure after 32 days of continuous sliding. ${ }^{36}$

Hydrogen plays an important role in determining the tribological properties of a-C:H film. Hydrogen can have the following effects on the internal structure of a-C:H film. ${ }^{24}$ (1) a$\mathrm{C}: \mathrm{H}$ containing ample hydrogen in their interiors and on their surfaces have little or no dangling $\sigma$ bonds, which can cause strong adhesion during sliding. Since surfaces containing $\mathrm{C}-\mathrm{H}$ bonds are chemically inert, adhesion is avoided between the film's surface and its counterpart (or between the film's surface and the surface of the transfer layer). Meanwhile, unbound hydrogen atoms or molecules can eliminate the $\sigma$ bonds formed by thermal desorption or mechanical effects, reducing the adhesion between the film and its counterpart and leading to a lower COF. (2) Partial carbon atoms (or carbon atoms at the film's surface) can become di-hydrogenated such that each surface carbon chemically bonds with two hydrogen atoms. Dihydrogenated carbon increases the surface's hydrogen density and better protects or passivates the surface, resulting in ultralow friction.

Obviously, these mechanisms only include contributions from the internal structure of a-C:H on the film's tribological behavior while ignoring the effect of the atmosphere. According to this standard interpretation, a-C:H films should display the same tribological behaviors in inert environment (dry $\mathrm{N}_{2}$, dry Ar, or ultra-high vacuum).

\section{Materials and methods}

\subsection{Deposition of a-C:H films}

The a-C:H films about $0.5 \mu \mathrm{m}$ thick were deposited on Si (100) wafers and steel ball by a plasma enhanced chemical vapor deposition (PECVD) technique, using $\mathrm{CH}_{4}$ plus $\mathrm{H}_{2}$ as the feedstock. A base pressure of $3.0 \times 10^{-3} \mathrm{~Pa}$ was attained in the chamber with a turbomolecular pumping system. Prior to deposition, the substrates were cleaned with Ar plasma sputtering at a bias voltage of $-400 \mathrm{~V}$ for $15 \mathrm{~min}$ so as to remove the native oxide on the Si surface or steel ball. Then the source gases were introduced into the deposition chamber and the formation of a-C:H films was commenced. Typical gas pressure during the film deposition was $5 \mathrm{~Pa}$, and RF power was $100 \mathrm{~W}$. The deposition conditions of a-C:H are as follows: (1) applied dc bias of $200 \mathrm{~V}$, (2) rf power of $100 \mathrm{~W}$, (3) gas flow rates of $\mathrm{CH}_{4}=7.3$ SCCM and $\mathrm{H}_{2}=20 \mathrm{SCCM}$, (4) deposition pressure of $5 \mathrm{~Pa}$, and (5) deposition time of $3 \mathrm{~h}$. For the a-C:H coated steel balls, the balls first coated with a 50-70 $\mathrm{nm}$ thick silicon bond layer by sputtering $\mathrm{Si}$ from a target. Then, $\mathrm{CH}_{4}$ and $\mathrm{H}_{2}$ source gases were bled into the chamber and the deposition of a-C:H on steel balls was started. The deposition conditions are same as on the $\mathrm{Si}$ surface. The a-C:H film show typical diamond-like carbon characteristics, with high hardness, extreme elasticity, superlow friction, etc. The detail properties of the films were published in our recent paper. ${ }^{37-40}$

\subsection{Friction tests}

The friction and wear behaviors of the resulting films sliding against a-C:H coated steel balls and uncoated $\mathrm{Si}_{3} \mathrm{~N}_{4}$ balls (diameter $4 \mathrm{~mm}$, density $3.2 \mathrm{~g} \mathrm{~mm}^{-3}$, hardness 1400-1700 GPa, surface roughness $0.025-0.032 \mathrm{~mm}$ ) were evaluated on a ballon-disk test rig equipped with an environmental chamber with which the relative humidity and gaseous environment could be controlled. The friction tests were performed in dry $\mathrm{N}_{2}$, dry Ar and vacuum, respectively, at a normal load of $2 \mathrm{~N}$, a sliding velocity of about $125 \mathrm{~m} \mathrm{~min}^{-1}$, to a maximum sliding duration of $60 \mathrm{~min}$.

\subsection{Model construction and calculations}

A single diamond cubic unit cell has five layers of carbon atoms along its $z$-axis. In order to simplify the model, three adjacent layers of carbon atoms, including the (001) plane, were selected. Since carbon atoms in the (001) plane contain two dangling bonds, a reconstruction was needed to improve the structure of the (001) surface. By connecting adjacent atoms with single bonds and adjusting the bond length to $1.60 \AA$, the carbon atoms on the (001) plane were limited to a single dangling bond. The bond length between the first and second layers of carbon atoms in the model was $1.61 \AA$. Bond lengths of $1.60 \AA$ and 1.61 $\AA$ were selected so that the surface reconstruction formed $\mathrm{C}-\mathrm{C}$ single bonds with similar lengths in the first and second layers. The single bond length between the second and third layer of carbon atoms in the model was $1.54 \AA$, which was consistent with the standard bond length of diamond. All dangling carbon bonds were saturated with hydrogen atoms such that the final 
model was composed of 40 carbon atoms and 52 hydrogen atoms. Since the model was relatively large and full optimization for this kind of model usually leads to severe deformation, the model was optimized locally with Gaussian 98. Only the hydrogen atoms bonded to carbon atoms at the (001) surface were optimized, leading to a hydrocarbon bond length of $1.08 \AA$. The configurations of the remaining model remained unchanged. B3LYP/6-31G* was used to calculate one-point energies. The energy of the hydrogen-saturated model surface was obtained. The other models used the same calculation method and basis set.

\section{Results and discussion}

\subsection{Tribological behaviors of a-C:H film}

As we all known, the tribological behaviors of a-C:H film are much different in dry $\mathrm{N}_{2}$, dry Ar, or ultra-high vacuum (Table 1 ). $\mathrm{a}-\mathrm{C}: \mathrm{H}$ film is known to maintain long-term superlubricity in dry $\mathrm{N}_{2}$ gas environments, while it has a very short wear life under ultra-high vacuum. For example, the a-C:H film deposited by Fontaine displayed a wear life of only 500 times under ultrahigh vacuum. ${ }^{34}$ Wang et al. reported similar results, and their a-C:H film had a very high COF (0.65) and a very short wear life (100 s) at $0.05 \mathrm{~Pa}$. However, the film's COF was drastically reduced to 0.012 in a $\mathrm{N}_{2}$ gas environment at $100 \mathrm{~Pa}^{14}$

The lubrication failure of a-C:H films in ultra-high vacuum is commonly believed to result from the generation of dangling surface bonds by the desorption of surface hydrogen. ${ }^{8}$ Under friction, the generated hydrocarbon compound can transfer to the surface of counterpart, effectively reducing the resistance to sliding. However, the hydrogen content of a-C:H film gradually decreases, because of the continuous loss of hydrogen atoms. An insufficient $\mathrm{C}-\mathrm{H}$ layer remains to provide a lubrication effect and avoid adhesion between the film and its counterpart, allowing cold welding to occur between the layers. This results in lubricant failure and the very short wear lives of a-C:H films.

$\mathrm{N}_{2}$ is believed to has two primary effects on the sliding surface that are related its chemical inertness. (1) $\mathrm{N}_{2}$ can prevent reactions of other molecules such as water and $\mathrm{O}_{2}$. Decreasing the partial pressures of $\mathrm{O}_{2}$ and water vapor does not impact the formation of a stable hydrocarbon polymer-like layer between the friction interfaces, as has been previously confirmed. ${ }^{41,42}$ (2) Passivation the carbon dangling bonds due to the loss of hydrogen atoms during friction process, and prevent chemical interactions between the surface and its counterpart, which can retard chemical interactions between the surface and its counterpart. During friction, the surface's $\mathrm{C}-\mathrm{H}$ bonds are broken and dangling carbon bonds are formed. $\mathrm{N}_{2}$ combines with these dangling carbon bonds and prevents the formation of dangling surface bonds, maintaining the surface's ultra-low COF and wear life.

However, it seems that the aforementioned explanations require additional consideration. First, the bond energy of $\mathrm{C}-\mathrm{H}$ is higher than that of $\mathrm{C}-\mathrm{C}$, indicating the higher chemical stability of the $\mathrm{C}-\mathrm{H}$ bond. This has been shown experimentally by the inability to break $\mathrm{C}-\mathrm{H}$ bonds below $700{ }^{\circ} \mathrm{C} .^{\mathbf{2 4 , 4 5}}$ Therefore, it is unlikely that $\mathrm{C}-\mathrm{H}$ bonds are broken on the surface of $\mathrm{a}-\mathrm{C}: \mathrm{H}$ films that are under friction (usually below $700{ }^{\circ} \mathrm{C}$ ). Moreover, according to the above explanation, hydrogen-free carbon films (e.g., a-C and ta-C) should have a low COFs in dry $\mathrm{N}_{2}$ gas environments. This is because the three carbon bonds in a hydrogen-free carbon film can form $\sigma$ bonds with adjacent carbon atoms, but the fourth bond is free and present on the surface. Therefore, the free bonds become saturated with $\mathrm{H}_{2} \mathrm{O}$ and $\mathrm{O}_{2}$ in air, forming dangling carbon bonds on the surface due to desorption of these adsorbed substances during friction processes. ${ }^{24}$ Assuming the above explanation is correct, $\mathrm{N}_{2}$ molecules can also combine with these dangling carbon bonds, reducing the surface energy of the film and further decreasing the COF. However, a-C and ta-C films have very high and unstable COFs in dry $\mathrm{N}_{2}$ gas environments. ${ }^{27}$ Therefore, these explanations are called into question.

In addition, although $\mathrm{Ar}$ and $\mathrm{N}_{2}$ are both chemically inert, the tribological behaviors of a-C:H films are different in these two atmospheres. ${ }^{33,35,44}$ Usually, a-C:H films have higher COFs in dry Ar gas environment (Table 1). Fontaine et al. found that the wear life of a-C:H film under vacuum is very short, only 40 times. Ar gas environments do not obvious improve the tribological behaviors of a-C:H films, and the wear life of a-C:H film in $\mathrm{Ar}$ gas environment at $1 \mathrm{kPa}$ is only approximately 270 times. $^{34} \mathrm{Ji}$ et al. prepared a-C:H film containing many $\mathrm{sp}^{2}$ hybridized carbon rings. This film had a COF of 0.005 to 0.006

Table 1 Summary of reported friction coefficient of a-C:H and doped-DLCs at dry $\mathrm{N}_{2}, \mathrm{Ar}$ and UHV

\begin{tabular}{|c|c|c|c|c|}
\hline & Dry $\mathrm{N}_{2}$ & Dry Ar & Ultra-high vacuum (UHV) & Reference \\
\hline \multirow[t]{6}{*}{ a-C:H } & 0.003 & - & - & Erdemir et al..$^{36}$ \\
\hline & 0.005 & 0.010 (sliding distance only $30 \mathrm{~m}$ ) & - & Ji et $a l .{ }^{35}$ \\
\hline & - & - & 0.005 (wear life only $900 \mathrm{~s}$ ) & Li et $a l .{ }^{17}$ \\
\hline & 0.03 & 0.04 & - & Erdemir et $a .^{41}$ \\
\hline & - & - & 0.18 (sliding distance less than $5 \mathrm{~m}$ ) & Meunier et $a l^{11}$ \\
\hline & - & - & $\sim 0.1$ (wear life only 40 cycles) & Wang et al. ${ }^{43}$ \\
\hline $\mathrm{a}-\mathrm{C}: \mathrm{H}: \mathrm{Si}$ & 0.001 & 0.016 & - & Chen et $_{\text {al. }}^{33}$ \\
\hline a-C:H:Si:O & - & - & 1.2 (wear life only 15 cycles) & Koshigan et al. ${ }^{44}$ \\
\hline
\end{tabular}


in dry $\mathrm{N}_{2}$ gas atmosphere, lower than that in Ar gas atmosphere (0.01). Additionally, the film's wear life is much lower in Ar gas atmosphere than in dry $\mathrm{N}_{2}$ gas atmosphere. ${ }^{35}$ These observations indicate that the tribological behaviors of a-C:H film are very similar in Ar gas atmosphere and under vacuum. Both environments produce very short wear lives. The COF of a$\mathrm{C}: \mathrm{H}: \mathrm{Si}$ in $\mathrm{N}_{2}$ gas atmosphere can be as low as 0.001 , while it is as high as 0.016 in Ar gas atmosphere. ${ }^{33}$ Clearly, these experimental observations are not explained by the existing tribological mechanisms. Our experiments were similar with the reported tribological behavior of a-C:H film (Fig. 1). The film displays stable and super-low friction in dry $\mathrm{N}_{2}$ gas environments, the average COF is about 0.005, while the COF is higher in dry Ar gas than in dry $\mathrm{N}_{2}$ gas, the average COF is about 0.026 in dry Ar gas. However, the film shows much high and unstable friction in vacuum, the average COF is about 0.172 .

Sliding speed also greatly affects the COF of a-C:H film. Heimberg et al. studied the effect of sliding speed on the run-in stage (the first 20 times) tribological behavior of a-C: $\mathrm{H}$ film in $\mathrm{N}_{2}$ gas atmosphere. Sliding speed determines the exposure time of the wear surface to the atmosphere. When the exposure time is changed, a-C:H film's ultra-low friction can be maintained, suppressed, and recovered, ${ }^{46}$ indicating that $\mathrm{N}_{2}$ physically interacts with the surface of the a-C:H film and forms a physically adsorbed layer. Since the surface of a-C:H film is known to be composed of $\mathrm{C}-\mathrm{H}$ bonds, $\mathrm{N}_{2}$ molecules must physically adsorb to these surface $\mathrm{C}-\mathrm{H}$ bonds. Even if the adsorbed $\mathrm{N}_{2}$ layer desorbs under mechanical shear during sliding, other $\mathrm{N}_{2}$ molecules can take their place and form a newly adsorbed layer, further altering the tribological behavior of the a-C:H film. Following this hypothesis, Ji et al. investigated the effect of exposure time on the friction properties of a-C:H films in three different atmospheres (dry $\mathrm{N}_{2}, \mathrm{CO}_{2}$, and Ar). ${ }^{35}$ They found that the COFs of a-C:H films in dry $\mathrm{N}_{2}$ and $\mathrm{CO}_{2}$ followed the same variation rule with exposure time such that an increase in exposure time caused the COF to first decrease and then increase. However, the COF of the a-C:H film in dry Ar gas

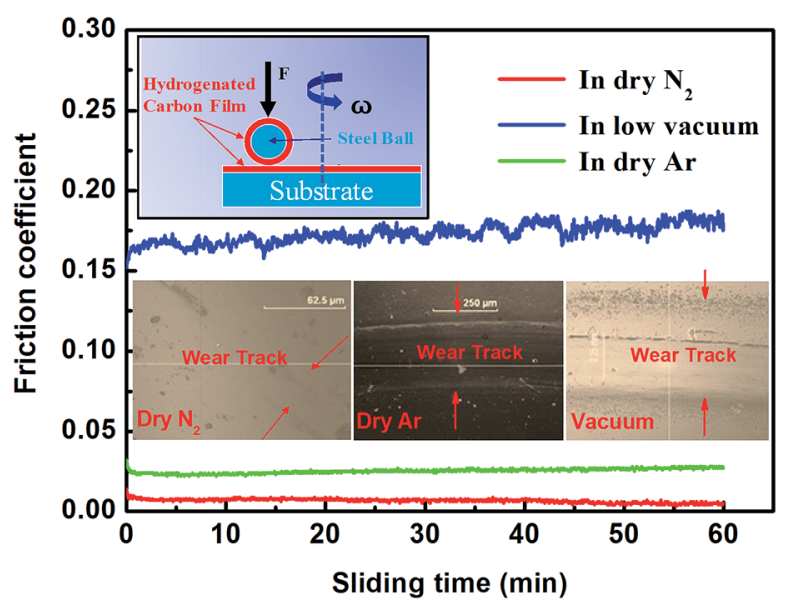

Fig. 1 Hydrogenated carbon (a-C:H) films against a-C:H coated steel ball in dry $\mathrm{N}_{2}$, dry Ar and vacuum, inset are the optical pictures of wear tracks on the a-C:H films at the three environment. atmosphere was almost unaffected by exposure time, ${ }^{35}$ similar to the results reported by Fontaine.

These phenomena indicate that different gases have different effects on adsorption at the surfaces of a-C:H films. $\mathrm{N}_{2}$ and $\mathrm{CO}_{2}$ are adsorbed rapidly on the surfaces of a-C:H films, allowing them to participate in tribochemical reactions and further affect the tribological behavior of the films. However, $\mathrm{Ar}$ hardly adsorbs on the surface of a-C:H films, making the film's tribological behavior similar in both $\mathrm{Ar}$ and under vacuum. $\mathrm{Ar}$ has only a very limited effect on the film's tribological behavior.

\subsection{Hydrogen bond-like mechanism}

These studies clearly indicate that interactions at the gas-solid interfaces of a-C:H films can occur during friction processes, and these interactions are the main cause of fluctuations in tribological behavior. However, most studies have only examined the tribophysical and tribochemical reactions occurring during friction processes and have ignored the characteristics and electronic properties of the environmental gas molecules $\left(\mathrm{N}_{2}, \mathrm{Ar}\right.$, etc.). Overlooking these features has limited our understanding of the friction mechanisms of a-C:H films. It is important to understand why Ar is not adsorbed on a-C:H film's surface while $\mathrm{N}_{2}$, a different inert gas, is easily adsorbed on the same film. It is also unclear why the adsorption of $\mathrm{N}_{2}$ significantly reduces the $\mathrm{COF}$ of a-C:H film. Unfortunately, existing tribological mechanisms do not explain these phenomena. To solve these problems, we accounted for the properties of the environmental molecules, such as their internal electronic structure, their electronegativity, the surface bonding state of a$\mathrm{C}: \mathrm{H}$ film, and synergistic effects between these factors.

As discussed above, the surface of a-C:H film is mainly composed of $\mathrm{C}-\mathrm{H}$ passivated bonds in which an electron from hydrogen combines with an electron from carbon to form a covalent $\mathrm{C}-\mathrm{H}$ bond. ${ }^{24}$ However, carbon is more electronegative than hydrogen, causing the bonded electron pairs between carbon and hydrogen to be closer to the carbon atom. The migration of bonded electron pairs results in a nearly empty electron orbital on the hydrogen atom, which is in an electrondeficient state, and easy to form hydrogen bonds with multielectron atom or group of atoms.

As we known, a $\mathrm{N}_{2}$ molecule contains two nitrogen atoms. In its electronic structure, nitrogen contains three pairs of electrons that contribute to forming chemical bonds, two $\pi$ bonds and one $\sigma$ bond. Therefore, each nitrogen atom has a pair of unbonded electrons (: $\mathrm{N} \equiv \mathrm{N}$ :) (Fig. 2). However, nitrogen is very electronegative. When $\mathrm{N}_{2}$ molecules get close to the surface of a$\mathrm{C}: \mathrm{H}$ film, charge attraction occurs between the hydrogen atoms in the surface's $\mathrm{C}-\mathrm{H}$ bonds and the electronegative $\mathrm{N}_{2}$ molecules. In other words, a lone electron pair of the $\mathrm{N}_{2}$ molecule interacts with the empty orbital of the bonded hydrogen atom, thus forming a mutual interaction (i.e. $-\mathrm{C}-\mathrm{H} \cdots: \mathrm{N} \equiv \mathrm{N}$ :). This effect is similar to hydrogen bonding between water molecules (Fig. 2). Hydrogen bonds are usually weak interactions between electron-deficient hydrogen atoms and electron-rich atoms or group of atoms which are typically weaker than chemical bonds but stronger than intermolecular forces (van der Waals forces). 


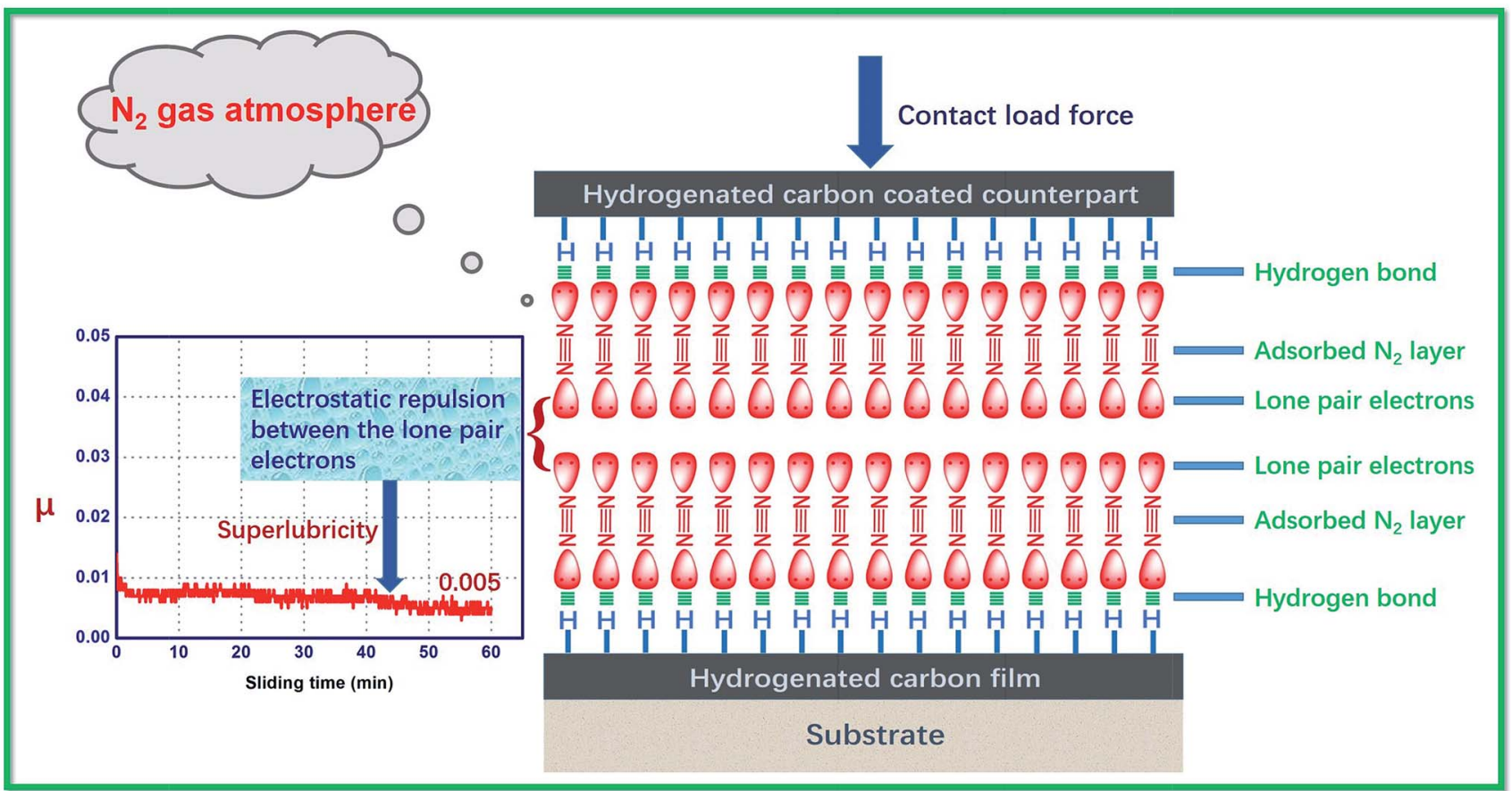

Fig. 2 Schematic diagram of tribological mechanisms of a-C:H films in dry $\mathrm{N}_{2}$ gas atmosphere.

Therefore, $\mathrm{N}_{2}$ molecules can easily adsorb on the surface of a$\mathrm{C}: \mathrm{H}$ film. This adsorption is relatively weak, allowing shear stress during the friction process to cause $\mathrm{N}_{2}$ desorption.

\subsection{Electronic interactions mechanism}

Although $\mathrm{N}_{2}$ molecules are easily adsorbed on the surfaces of a$\mathrm{C}: \mathrm{H}$ films, it remains unclear why the adsorption of $\mathrm{N}_{2}$ significantly reduces the films' COFs. To illustrate the problem, it is helpful to consider the electronic structure of $\mathrm{N}_{2}$ molecule. As mentioned above, a lone pair of electrons can interact with the hydrogen atoms at the surface of a-C:H film, causing adsorption effects that are similar to hydrogen bonds. In this case, the other lone pair of electrons remains. For the self-mated sliding of a-C:H film in $\mathrm{N}_{2}$ gas atmosphere, the film and its counterpart both adsorb a layer of $\mathrm{N}_{2}$ molecules (Fig. 2). As the two surfaces move close to each other, the lone pairs of electrons are near each other as well. The interaction between lone pairs of electrons leads to dipole interactions between the contacting surface, which increase the electrostatic repulsion between the contacting surfaces (Fig. 2). This electrostatic repulsion results in the super-low friction of the a-C:H film in dry $\mathrm{N}_{2}$ gas environment (Fig. 2).

However, no gas molecules are adsorbed under ultra-high vacuum, eliminating this source of electrostatic repulsion. Because of this, a-C:H films have higher COFs under ultra-high vacuum. For Ar molecule, which is composed of a single $\mathrm{Ar}$ atom with a saturated outer electronic orbital and a stable electron cloud structure. Therefore, electrons from an $\mathrm{Ar}$ molecule do not interact with those in the surface of an a-C:H film, limiting the adsorption of Ar on the surfaces of a-C:H films. A small amount of Ar might adsorb on the surface of a-
C:H film through van der Waals forces, but electrostatic repulsion does not occur between the two adsorption layers because of Ar's stable electron cloud structure. So the Ar gas environment is similar to vacuum. The major difference between these two environments is their thermal conductivities. Under ultrahigh vacuum, the temperatures of the contacting surfaces are approximately $100-200{ }^{\circ} \mathrm{C}$ higher than in dry inert gas atmosphere, ${ }^{44}$ perhaps causing the shorter wear life of a-C:H films under ultra-high vacuum than in dry Ar gas environments.

Although the COF of a-C:H film sliding against other material (e.g., $\mathrm{Fe}, \mathrm{Al}_{2} \mathrm{O}_{3}, \mathrm{Si}_{3} \mathrm{~N}_{4}$, or $\mathrm{SiC}$ ) is relatively high compared to that of self-mated sliding, the effect of the environmental atmosphere on the tribological behavior of $\mathrm{a}-\mathrm{C}: \mathrm{H}$ film is the same under these different conditions, please see our previous works ${ }^{47-52}$ i.e., the COF is lower in dry $\mathrm{N}_{2}$ gas than in other atmospheres (Fig. 3). The wear track is deep and wide, indicating heavy adhesive wear was happened during sliding under vacuum. This clear shows that the effect of the atmosphere is not dependent on the counterpart's material.

Since a-C:H is a polymer-like hydrocarbon compound, it contains weak bonds $\left(\sim 8 \mathrm{~kJ} \mathrm{~mol}^{-1}\right)$ between its hydrogenated carbon chains. $^{53}$ Therefore, the polymer-like a-C:H easily transfers to the surface of the counterpart during the friction process and forms a transfer layer. Erdemir et al. and our group have confirmed that this transfer layer is mainly composed of hydrogenated carbon ions $\left(\mathrm{CH}^{-}, \mathrm{CH}_{2}{ }^{-}\right.$, and $\left.\mathrm{C}_{2} \mathrm{H}^{-}\right)$rather than graphite. ${ }^{15,30}$ Therefore, the transfer layer is considered to be a nonstoichiometric hydrocarbon $\left(\mathrm{CH}_{x}\right)$, and the $\mathrm{C}-\mathrm{H}$ bond has a high chemical stability. The $\mathrm{C}-\mathrm{H}$ bonds of the $\mathrm{CH}_{x}$ transfer layer are not believed to break during the friction process. Based on this assumption, the $\mathrm{CH}_{x}$ transfer layer has two effects on the 


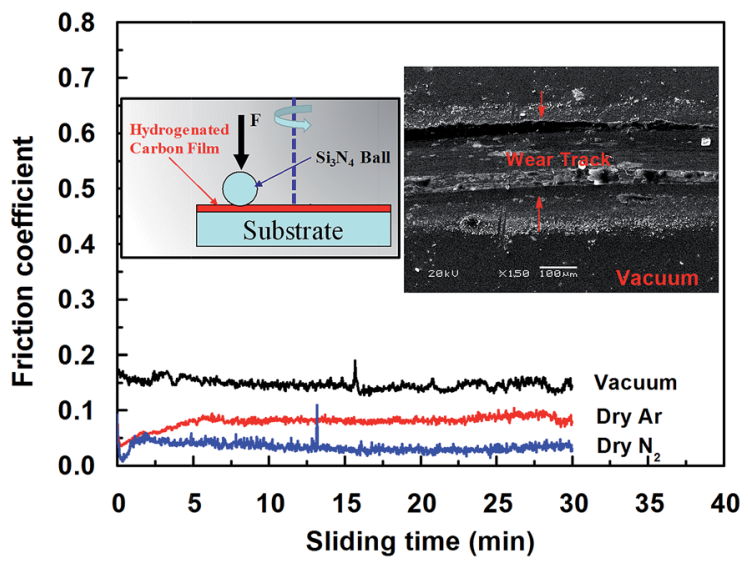

Fig. 3 a-C:H films against uncoated $\mathrm{Si}_{3} \mathrm{~N}_{4}$ ball in dry $\mathrm{N}_{2}$, dry $\mathrm{Ar}$ and vacuum, inset are the wear tracks on the a-C:H films under vacuum.

friction process. (1) The lower shear stress of the $\mathrm{CH}_{x}$ transfer layer effectively prevents direct contact between the film and its counterpart, further reducing friction. (2) The $\mathrm{CH}_{x}$ transfer layer also adsorbs $\mathrm{N}_{2}$ molecules and forms hydrogen bond-like interactions, which generate dipole and electrostatic repulsion at the friction interface. Both of these effects lead to a very low $\mathrm{COF}$ in $\mathrm{N}_{2}$ gas atmosphere.

Unfortunately, it is very difficult to verify these hypotheses using in situ on-line monitoring techniques for gas adsorption and electron interactions at the interface of a-C:H film. From a tribological perspective, the friction between two surfaces is mainly determined by the chemical and physical interactions between the contacting surfaces and the characteristics and extent of the chemical interactions between the friction interface and the environment.

So theoretical calculations are necessary to understand atomic scale physical and chemical interactions. Molecular dynamics simulations and density functional theory have been broadly applied for tribological studies of carbon-based films (e.g., diamond, a-C:H, and a-C). ${ }^{54-59}$ However, these studies have mainly focused on the effects of $\mathrm{H}$-passivated and $\mathrm{OH}^{-}$ passivated surfaces on the tribological behavior of carbon films and the theoretical calculation of interactions between carbon films and counterpart materials. ${ }^{53-59}$ Studies of the effects of gas adsorption on the tribological behavior of carbonbased films are less common. Wang et al. calculated the effect of $\mathrm{O}_{2}$ adsorption on the tribological behaviors of diamond and a-C films using $a b$ initio principles..$^{59}$ To the best of our knowledge, no one has determined the effect of gas adsorption on the tribological behavior of a-C:H film using theoretical calculations.

In this work, diamond was simulated using density functional theory, and the dangling carbon bonds on the diamond surface were saturated with hydrogen to produce a-C:H film. Base on the model of a-C:H, the model of a-C:H in $\mathrm{N}_{2}$ gas atmospheres was constructed. The differences in the friction forces were analyzed between two surfaces during self-mated sliding as a function of configuration and interlayer distance.

\subsection{Density functional simulation}

3.4.1 Model of hydrogen-saturated carbon (a-C:H). DLC films have amorphous carbon structures and are composed of three-dimensional intersecting carbon-ring networks formed of covalent bonds. The main bonding types of film are $\mathrm{sp}^{3}$ (diamond) and $\mathrm{sp}^{2}$ bond (graphite). ${ }^{6}$ Generally, a higher $\mathrm{sp}^{3} \mathrm{C}-\mathrm{C}$ content produces a harder and more compact film that has diamond-like macroscopic properties, making it difficult to construct a realistic DLC film model. Therefore, we constructed the DLC thin film model using a reasonable reconstruction of diamond. Base on this, we built the a-C:H (Model A, Fig. 4A).

Model A was flipped along its $z$-axis and re-imported. The distance between the two parts was adjusted along the $z$-axis. The relative hydrogen-hydrogen distance between the two layers, i.e., the hydrogen-hydrogen internuclear distance, is referred to as $d_{\mathrm{H}-\mathrm{H}}$. The interaction model for the hydrogensaturated a-C:H film was constructed (Fig. 4B). This model was equivalent to the self-mated sliding of a-C:H film under ultra-high vacuum. The value of $d_{\mathrm{H}-\mathrm{H}}$ was maintained between $1.50 \AA$ and $4.00 \AA$. This distance range was based on approximate atomic sizes for this chemical structure. Excessively large or short distances would be unreasonable. The calculation produced a similar result, a short distance leads to a sharp rise in energy, while a large distance leads to no significant change in energy. A step size of $0.10 \AA$ was used. The single point energy, $E_{\mathrm{H}}$, of the complex system was calculated at each distance.

The energy difference between the complex system in Model $\mathrm{B}$ and the two sub-systems in Model A were expressed as $\Delta E_{\mathrm{H}}=$ $E_{\mathrm{H}}-2 E_{\mathrm{a}}$. A plot of $\Delta E_{\mathrm{H}} v s . d_{\mathrm{H}-\mathrm{H}}$ is shown in Fig. 5A. An increase in $d_{\mathrm{H}-\mathrm{H}}$ caused the fluctuations in $\Delta E_{\mathrm{H}}$ to gradually decrease. At a $d_{\mathrm{H}-\mathrm{H}}$ of $2.60 \AA, \Delta E_{\mathrm{H}}$ reached a minimum of -0.0223 Hartree $(-0.6068 \mathrm{eV})$, indicating that the model had reached its most stable state. In this case, the interlayer distance, $l_{\mathrm{H}-\mathrm{H}}$, was obtained by subtracting $2 r_{\mathrm{H}}$ from $d_{\mathrm{H}-\mathrm{H}}$. The van der Waals radius of the hydrogen atoms was $1.20 \AA$, so $l_{\mathrm{H}-\mathrm{H}}$ of the most stable configuration was $0.20 \AA$.

3.4.2 Model of surface $\mathbf{N}_{2}$-adsorbed a-C:H. Using surface hydrogen-saturated Model A (a-C:H) as a basis, $\mathrm{N}_{2}$ molecules were adsorbed along the vertical hydrogens along the (001) carbon atoms with a nitrogen-nitrogen bond length of $1.10 \AA$ A. The distance between adjacent $\mathrm{N}_{2}$ molecules was no less than the sum of the van der Waals radii of the nitrogen atoms (3.10

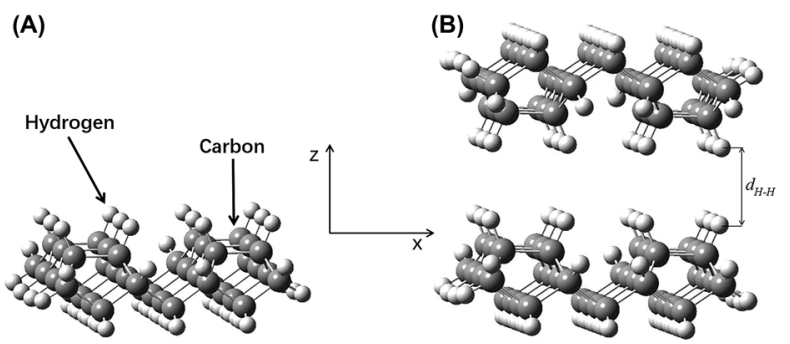

Fig. 4 (A) Model construction of a-C:H (Model A). (B) The complex system (Model B). 


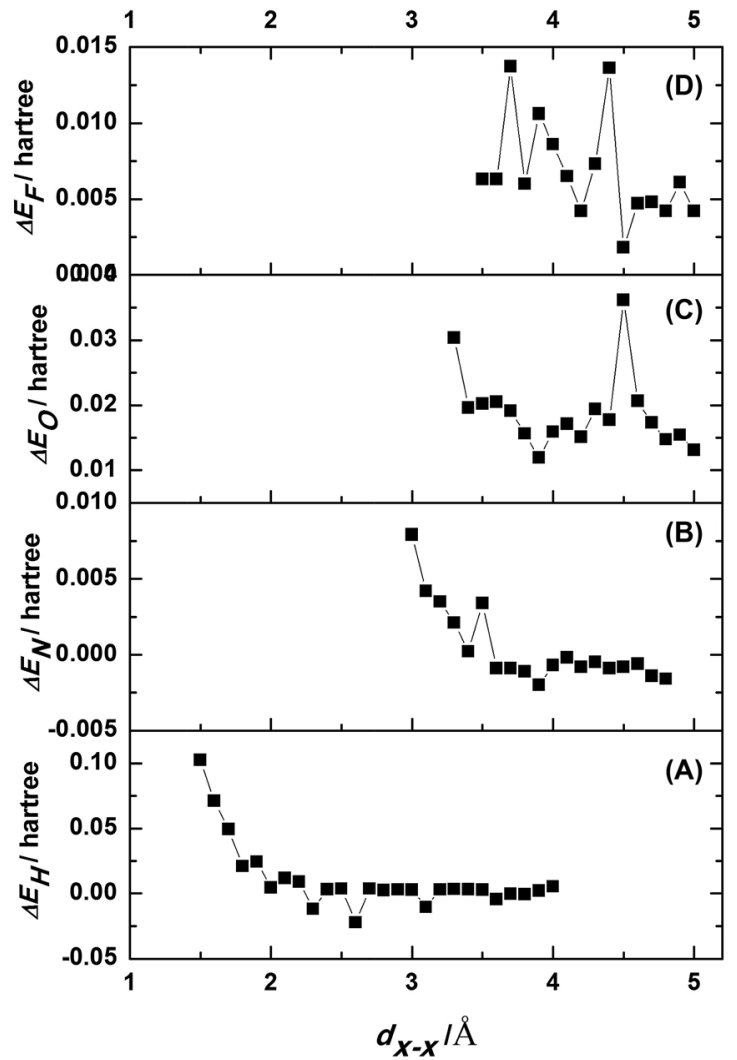

Fig. 5 A plot of (A) $\Delta E_{\mathrm{H}} v s . d_{\mathrm{H}-\mathrm{H}}$ (B) $\Delta E_{\mathrm{N}} v s . d_{\mathrm{N}-\mathrm{N}}$ (C) $\Delta E_{\mathrm{O}} v s . d_{\mathrm{O}-\mathrm{O}}$ (D) $\Delta E_{\mathrm{F}} v s . d_{\mathrm{F}-\mathrm{F}}$.

A). Six $\mathrm{N}_{2}$ molecules were adsorbed. The sum of the radii of the nitrogen and hydrogen atoms $(2.75 \AA)$ was used as the distance between nitrogen and hydrogen. Only the bond lengths of the $\mathrm{N}_{2}$ molecules were optimized to obtain the $\mathrm{N}_{2}$-adsorbed a-C:H model (Fig. 6A). The energy of Model C, $E_{\mathrm{c}}$, was obtained using single-point energy calculations. The $\mathrm{N}_{2}$-adsorbed complex model is model $\mathrm{D}$ (Fig. 6B), and the relative $\mathrm{N}-\mathrm{N}$ internuclear distance is referred to as $d_{\mathrm{N}-\mathrm{N}}$. The value of $d_{\mathrm{N}-\mathrm{N}}$ was changed from $3.00 \AA$ to $4.80 \AA$ with a step size of $0.10 \AA$. The energy at every point of the double-layer complex, $E_{\mathrm{N}}$, was calculated.

The energy differences between Model D and two subsystems of Model C were expressed as $\Delta E_{\mathrm{N}}=E_{\mathrm{N}}-2 E_{\mathrm{c}}$. A plot
(A)

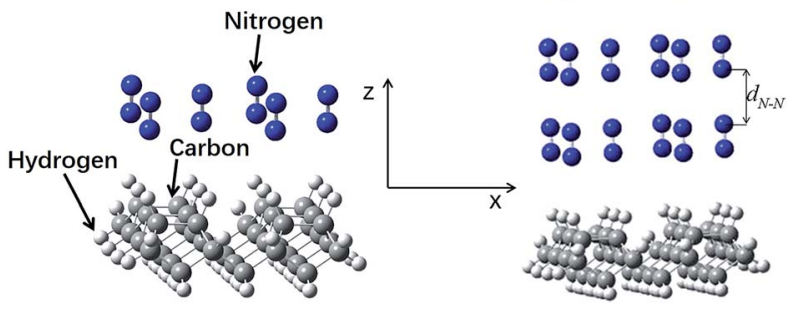

Fig. 6 (A) Model construction of $\mathrm{N}_{2}$-adsorbed a-C:H (Model C). (B) The complex system (Model D). of $\Delta E_{\mathrm{N}} v s . d_{\mathrm{N}-\mathrm{N}}$ is shown in Fig. 5B. An increase in $d_{\mathrm{N}-\mathrm{N}}$ caused the fluctuations in $\Delta E_{\mathrm{N}}$ to gradually decrease. When $d_{\mathrm{N}-\mathrm{N}}$ was $3.90 \AA, \Delta E_{\mathrm{N}}$ reached a minimum of -0.0020 Hartree $(-0.0544$ $\mathrm{eV}$ ), indicating that this was the model's most stable configuration. The van der Waals radius of nitrogen was $1.55 \AA$, and, therefore, $l_{\mathrm{N}-\mathrm{N}}$ was $0.80 \AA$.

3.4.3 Surface $\mathrm{O}_{2}$-adsorbed a-C:H model. Since $\mathrm{O}_{2}$ often participate tribochemical reactions at the interfaces of a-C:H films during friction processes, we also constructed the models of a-C: $\mathrm{H}$ in $\mathrm{O}_{2}$ atmospheres. Furthermore, fluoride is known to be an excellent solid lubricant, $\mathrm{F}$ doped a-C:H can improve the films' tribological properties, so we constructed the model of $\mathrm{F}$ doped a-C:H (a-C:F:H).

Since $\mathrm{O}_{2}$ is a relatively reactive gas molecule, tribochemical reactions occur readily at the surfaces of a-C: $\mathrm{H}$ films during friction processes. ${ }^{27}$ If $\mathrm{O}_{2}$ molecules were directly adsorbed on the surface of a-C:H film like the $\mathrm{N}_{2}$ molecules in Model $\mathrm{C}$, the system became very unstable, clearly indicating that $\mathrm{O}_{2}$ molecules do no directly adsorb on the surface of a-C:H in the same manner as $\mathrm{N}_{2}$ molecules. Many studies have clearly indicated that contacting a-C: $\mathrm{H}$ surfaces are oxidized in an $\mathrm{O}_{2}$ environment. On this basis, we developed adsorption Model $\mathrm{E}$ for $\mathrm{a}-\mathrm{C}: \mathrm{H}$ film in $\mathrm{O}_{2}$ environment by saturating the surface with oxygen bridges $(\mathrm{C}-\mathrm{O}-\mathrm{C})$. We removed the $\mathrm{H}$ atoms, which were connected to the $\mathrm{C}$ atoms on the (001) plane in Model A. Instead, the oxygen bridge connected these $\mathrm{C}$ atoms via single bonds. To generate more reasonable bridging bonds, the single bond length of the reconstructed $\mathrm{C}-\mathrm{C}$ bonds on the (001) plane were adjusted to $1.53 \AA$, and only the $\mathrm{C}-\mathrm{O}$ bond length was optimized. The optimized model is shown in Fig. 7A, where the $\mathrm{C}-\mathrm{O}$ bond length is $1.48 \AA$. The energy of Model F, $E_{\mathrm{f}}$, was obtained with single-point energy calculations. For the interlayer interactions in Model F (Fig. 7B), $d_{\mathrm{O}-\mathrm{O}}$ represents the relatively internuclear distance.

The value of $d_{\mathrm{O}-\mathrm{O}}$ was adjusted from $3.30 \AA$ to $5.00 \AA$ at a step size of $0.10 \AA$. The single-point energy of the complex system was calculated at each step. The energy difference between Model $\mathrm{F}$ and the two sub-systems of Model $\mathrm{E}$ is $\Delta E_{\mathrm{O}}=E_{\mathrm{O}}-2 E_{\mathrm{e}}$. A plot of $\Delta E_{\mathrm{O}} v s$. $d_{\mathrm{O}-\mathrm{O}}$ is shown in Fig. $5 \mathrm{C}$. At an oxygen internuclear distance of $3.90 \AA, \Delta E_{\mathrm{O}}$ reached a positive minimum value of 0.0119 Hartree $(0.3238 \mathrm{eV})$. The van der Waals radius of oxygen, $r_{\mathrm{O}}$, is $1.52 \AA$. So the interlayer distance, $l_{\mathrm{O}-\mathrm{O}}$, was $0.86 \AA$.

3.4.4 Partially fluorine-saturated a-C:H (F-doped a-C:H model). Fluoride is known to be an excellent solid lubricant. Many researchers have doped a-C:H films with fluorine to
(A)

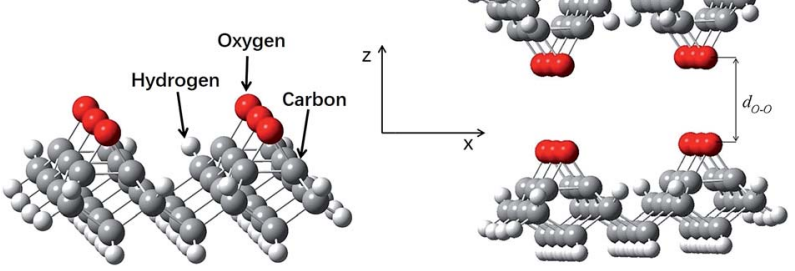

Fig. 7 (A) Model construction of $\mathrm{O}_{2}$-adsorbed a-C:H (Model E). (B) The complex system (Model F). 
improve the films' tribological properties. ${ }^{60}$ In a-C:F:H film, F directly connects to $\mathrm{C}$ to form a covalent $\mathrm{C}-\mathrm{F}$ bond. On the basis of the surface hydrogen-saturated Model A, Model G, which contained partially fluorine-saturated a-C:H, was constructed by substituting the six hydrogen atoms with fluorine atoms. Local optimization was applied in the model by fixing the other atoms and optimizing the $\mathrm{C}-\mathrm{H}$ and $\mathrm{C}-\mathrm{F}$ bond lengths on the (001) plane. The optimized model is shown in Fig. 8A, which contains a C-F bond length of $1.39 \AA$. The energy of Model G, $E_{\mathrm{F}}$, was also calculated.

The fluorine-fluorine internuclear distance in model $\mathrm{G}$ (Fig. $8 \mathrm{~B}$ ) is referred to as $d_{\mathrm{F}-\mathrm{F}}$, which was tested in the range from $3.50 \AA$ to $5.00 \AA$ at a step size of $0.10 \AA$. The energy of the double-layer at each point, $E_{\mathrm{F}}$, was calculated. The energy differences between the complex system in Model $\mathrm{H}$ and the two sub-systems of Model $\mathrm{G}$ were expressed as $\Delta E_{\mathrm{F}}=E_{\mathrm{F}}-2 E_{\mathrm{g}}$. A plot of $\Delta E_{\mathrm{F}} v s$. $d_{\mathrm{F}-\mathrm{F}}$ is shown in Fig. $5 \mathrm{D}$. At $d_{\mathrm{F}-\mathrm{F}}=4.50 \AA, \Delta E_{\mathrm{F}}$ reached its minimum value of 0.0018 Hartree $(0.0490 \mathrm{eV})$. Since the van der Waals radius of a fluorine atom is $1.47 \AA, l_{\mathrm{F}-\mathrm{F}}$ was $1.56 \AA$.

\subsection{Discussion}

All of the models had larger energy variations within the studied ranges and minimum $\Delta E$ values. The $\Delta E_{\min }$ values of Model $\mathrm{B}$ and Model D were negative at -0.0223 Hartree and -0.0020 Hartree $(-0.6068 \mathrm{eV}$ and $-0.0544 \mathrm{eV})$, respectively, while the $\Delta E_{\min }$ values of Models $\mathrm{F}$ and $\mathrm{H}$ were positive at 0.0018 Hartree and 0.0119 Hartree, $(0.0490 \mathrm{eV}$ and $0.3238 \mathrm{eV})$, respectively. The negative $\Delta E_{\min }$ values of the surface hydrogen-saturated and nitrogen-adsorbed a-C:H films indicated the formation of a double-layered system, which slightly decreased the system's total energy within a certain distance. Nitrogen adsorption can occur on the surface of a-C:H films in a manner that is consistent with experimental results. However, the formation of double-layered systems in the partially surface oxygen-saturated and fluorine-saturated a-C:H films always increased the system's total energy. These systems did not tend to form double layers. Under these artificial arrangements, repulsion reaches a minimum at the distance corresponding to the minimum energy difference. This likely resulted from the ability of $\mathrm{O}$ and $\mathrm{F}$ to directly bond with the carbon atoms in a$\mathrm{C}: \mathrm{H}$ film, altering the original internal structure of a-C:H and increasing the system's energy.

(A)

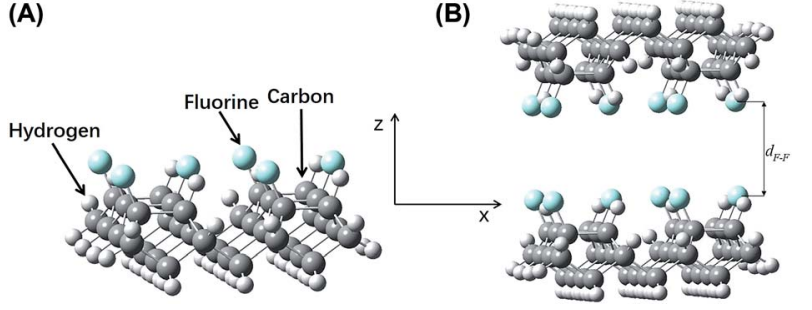

Fig. 8 (A) Model construction of fluorine-terminated a-C:H (Model G). (B) The complex system (Model H).
Charge repulsion is unstable when the same sign exists in all models. Meanwhile, dispersion forces add stability to the systems. However, because oxygen and fluorine are more electronegative, they produce stronger inter-layer repulsion, and their instability exceeds their stability. In the other models, there is less repulsion, allowing for a higher stability within a certain interlayer distance, perhaps explaining the positive and negative energy differences in the different models.

As shown in the energy difference curves of the different models, the energy minimums occur at different interlayer distances (Table 2), resulting in the different interlayer friction behaviours.

When considering friction forces, a greater distance between layers generally results in a weaker friction force. The interlayer friction force of the oxygen-bridged film is weaker than that of the hydrogen-saturated film. According to the above results, $l_{\mathrm{X}-\mathrm{X}}$ follows the order:

$$
l_{\mathrm{H}-\mathrm{H}}(0.20 \AA)<l_{\mathrm{N}-\mathrm{N}}(0.80 \AA)<l_{\mathrm{O}-\mathrm{O}}(0.86 \AA)<l_{\mathrm{F}-\mathrm{F}}(1.56 \AA)
$$

The friction force should be inversely proportional to distance, indicating that the environmental atmosphere is the main factor affecting the COF of a-C:H film. However, the COF of the a-C:H films in the four models did not follow this simple order. The four models were divided into two categories with Models B, D, and F in one category and Model $\mathrm{H}$ in the other. Models B, D, and F involve the self-mated sliding of a-C:H films in vacuum, nitrogen, and oxygen environments, respectively. Model $\mathrm{H}$ is of F-doped a-C:H film (a-C:F:H). Models D and F also have different surface structures. Oxygen molecules are not adsorbed on the surface of a-C:H film like nitrogen molecules are, but instead they can be incorporated into the surface of a$\mathrm{C}: \mathrm{H}$ as a surface oxygen bridge. In other words, the oxygen molecules oxidize the a-C:H film. During friction processes in a reactive atmosphere, a-C: $\mathrm{H}$ films inevitably experienced varying extents of oxidation, structural changes, and other tribochemical reactions. ${ }^{27,61}$ In the absence of oxygen, these processes can also occur through the adsorption of water vapor or organic compounds. Oxygen only acts as an oxidant, oxidizing the surface of carbon-based film, while water vapor (at low humidity) can be adsorbed at the friction interface and act as a molecular lubricant to reduce the solid-solid contact during friction processes. Water vapor at high humidity can lead to oxidation reactions at the interface and even act as an electrolyte to promote electrochemical reactions. ${ }^{61}$

Kim et al. reported that oxygen atmosphere had a smaller effect on the tribological behavior of a-C:H film than did an atmosphere containing water vapor. ${ }^{10}$ Although an increase in oxygen pressure increased the COF of a-C:H film, this increase was smaller than that caused by water vapor. ${ }^{10}$ At the same pressure, the COF of a-C:H film in oxygen atmosphere is lower than that in water vapor. Therefore, although the interlayer distance corresponding to a minimum energy difference in Model F was $0.86 \AA$, which was larger than those of Models A and $\mathrm{D}$, it was not shown that the COF of a-C:H film in oxygen was lower than that achieved under vacuum or in a nitrogen 
Table 2 The distance between the two surface of each model system ${ }^{a}$

\begin{tabular}{lllll}
\hline & Model B (a-C:H) & $\begin{array}{l}\text { Model D } \\
\left(\mathrm{N}_{2} \text { adsorbed a-C:H }\right)\end{array}$ & $\begin{array}{l}\text { Model F } \\
\left(\mathrm{O}_{2} \text { adsorbed a-C:H }\right)\end{array}$ & $\begin{array}{l}\text { Model H } \\
(\mathrm{F} \text { terminated a-C:H })\end{array}$ \\
\hline$d_{\mathrm{X}-\mathrm{X} / \AA} / \AA$ & 3.90 & 3.90 & 4.50 \\
$l_{\mathrm{X}-\mathrm{X} / \mathrm{A}}$ & 2.60 & 0.80 & 0.86 & 1.56 \\
$\Delta E /$ Hartree & 0.20 & -0.0020 & 0.0119 & 0.0018
\end{tabular}

${ }^{a} d_{\mathrm{X}-\mathrm{X}}$ is internuclear distance of the nearest two atoms layer, $\mathrm{X}$ represent the atomic element symbols; $l_{\mathrm{X}-\mathrm{X}}$ is the distance between the two layer, $l_{\mathrm{X}-\mathrm{X}}$ $=d_{\mathrm{X}-\mathrm{X}}-2 r_{\mathrm{X}}, r_{\mathrm{X}}$ is the Van der Waals radius of $\mathrm{X}$ atom.

environment. The reason is that oxidation can lead to structural changes in the surface of the a-C:H film, affect the formation of a $\mathrm{CH}_{x}$ transfer layer and influence the film's tribological behavior. To some extent, the calculations in this work reflect oxygen's relatively small effect on the tribological behavior of a$\mathrm{C}: \mathrm{H}$ film, which is consistent with experimental results.

As in Models B and D, Model D includes the adsorption of an additional nitrogen layer on the surface of Model B. This adsorbed nitrogen layer does not cause structural changes of the a-C:H film's surface. Therefore, the calculations for Models $\mathrm{B}$ and $\mathrm{D}$ can be directly compared. For Model D, the minimum energy difference occurred at an interlayer distance $0.80 \AA$, much greater than the $0.20 \AA$ of Model $\mathrm{B}$, indicating that the friction force was significantly reduced by the adsorbed nitrogen layer. As the calculation results clearly indicated, the COF of a-C:H film in a nitrogen atmosphere was much lower than that under vacuum, which was consistent with experimental results. ${ }^{11,14,17,33-36,41}$

Since fluorine can form chemical bonds with carbon, the Fdoped a-C:H film (Model $\mathrm{H}$ ) approximated the self-mated sliding of a-C:F:H under vacuum. The results indicated that the interlayer distance was $1.56 \AA$ when the partially fluorinesaturated Model $\mathrm{F}$ reached its minimum energy. This was significantly larger than the interlayer distances at which minimum energy differences were reached by the other three models, indicating that the interlayer friction force of the partially fluorine-saturated model was significantly reduced. These calculation results were expected, because F-doped a-C:H films have been relatively well studied and many researchers have prepared a variety of a-C:F:H films with excellent tribological properties. ${ }^{60}$ When two a-C:F:H films come into contact with each other, very strong electrostatic repulsion occurs between the surfaces because of fluorine's high electronegativity. Two a-C:F:H surfaces have higher repulsive forces than that of two a-C:H surfaces. In addition, a number of important known fluorine-containing compounds, such as polytetrafluoroethylene and perfluorinated C60, are known to be solid lubricating materials, suggesting that these results are credible and that the conclusions drawn from these theoretical simulations are reasonable.

\section{Conclusions}

The present work provides a mechanism to explain the role of $\mathrm{N}_{2}$ gas in decreases the COF of $\mathrm{a}-\mathrm{C}: \mathrm{H}$. We explored the tribological behavior of a-C:H films by accounting for the internal electronic structure of ambient gas molecules, their atomic electronegativity, the surface bonding state of the films, and the synergistic effects of these factors. Because of the unique electronic structure of the $\mathrm{N}_{2}$ molecule, which has two lone electron pairs, its lone-pair electrons interact with $\mathrm{C}-\mathrm{H}$ bonds on the surface of a-C:H films, forming $-\mathrm{C}-\mathrm{H} \cdots: \mathrm{N} \equiv \mathrm{N}$ : interactions that are similar to the hydrogen bonds in water. This causes $\mathrm{N}_{2}$ to be adsorbed on the film's surface. As two $\mathrm{N}_{2}$ adsorbed a-C:H surfaces approach each other, the other lonepair electrons in $\mathrm{N}_{2}$ become close, generating electrostatic repulsion and resulting in the very low COFs of films in dry $\mathrm{N}_{2}$ atmospheres. Meanwhile, Ar molecular contains one Ar atom and has a very stable electron cloud that prevents electronic interactions with the surface of a-C:H films. This also prevents Ar from adsorbing to the surfaces of a-C:H films. Under ultrahigh vacuum, the absence of adsorbed gas leads eliminates electrostatic repulsion. Therefore, a-C:H films under high vacuum and in Ar gas environment have high COFs and short wear lives.

Based on the above analysis, we constructed a-C:H film using density functional theory. Additional a-C:H models were constructed in different environments, including a-C: $\mathrm{H}$ with a surface layer of adsorbed $\mathrm{N}_{2}$ (to model $\mathrm{N}_{2}$ gas environment), a$\mathrm{C}: \mathrm{H}$ with saturated oxygen-bridges (C-O-C structures) on its surface (to model $\mathrm{O}_{2}$ gas environment), and partially fluorinesaturated a-C:H (to model fluorine doped a-C:H film). From different attributes like configuration and interlayer distance, friction forces were calculated between the two surfaces. These theoretical calculations matched experimental results, indicating that the proposed approach is reasonable.

\section{Acknowledgements}

The authors gratefully acknowledge the financial support by the National Natural Science Foundation of China (No. 51575253, 51562020, 51365026, and 51261014).

\section{References}

1 D. S. Grierson and R. W. Carpick, Nano Today, 2007, 2, 12-21.

2 A. Erdemir and C. Donnet, J. Phys. D: Appl. Phys., 2006, 39, R311-R327.

3 R. Zahid, H. H. Masjuki, M. Varman, R. A. Mufti, M. A. Kalam and M. Gulzar, Tribol. Lett., 2015, 58, 32.

$4 \mathrm{~J} . \mathrm{Xu}$ and J. Li, Friction, 2015, 3, 344-351. 
5 A. Erdemir and O. Eryilmaz, Friction, 2014, 2, 140-155.

6 J. Robertson, Mater. Sci. Eng., R, 2002, 37, 129-281.

7 A. Alazizi, A. J. Barthel, N. D. Surdyka, J. Luo and S. H. Kim, Friction, 2015, 3, 85-114.

8 J. Fontaine, T. Le Mogne, J. L. Loubet and M. Belin, Thin Solid Films, 2005, 482, 99-108.

9 W. Zhang, A. Tanaka, K. Wazumi and Y. Koga, Thin Solid Films, 2002, 413, 104-109.

10 H. I. Kim, J. R. Lince, O. L. Eryilmaz and A. Erdemir, Tribol. Lett., 2006, 21, 51-56.

11 C. Meunier, P. Alers, L. Marot, J. Stauffer, N. Randall and S. Mikhailov, Surf. Coat. Technol., 2005, 200, 1976-1981.

12 J. Andersson, R. A. Erck and A. Erdemir, Surf. Coat. Technol., 2003, 163-164, 535-540.

13 H. Okubo, R. Tsuboi and S. Sasaki, Wear, 2015, 340-341, 2-8. 14 L. Cui, Z. Lu and L. Wang, Carbon, 2014, 66, 259-266.

15 O. L. Eryilmaz and A. Erdemir, Surf. Coat. Technol., 2007, 201, 7401-7407.

16 F. Gao, A. Erdemir and W. T. Tysoe, Tribol. Lett., 2005, 20, 221-227.

17 Y. Wu, H. Li, L. Ji, Y. Ye, J. Chen and H. Zhou, Tribol. Int., 2014, 71, 82-87.

18 Y. Liu, A. Erdemir and E. I. Meletis, Surf. Coat. Technol., 1996, 86-87, 564-568.

19 J. D. Schall, G. Gao and J. A. Harrison, J. Phys. Chem. C, 2010, 114, 5321-5330.

20 D. Wang, S. Chang, Y. Huang, J. Wu, H. Lai and M. Leu, Carbon, 2014, 74, 302-311.

21 M. I. De Barros Bouchet, C. Matta, B. Vacher, T. Le-Mogne, J. M. Martin, J. von Lautz, T. Ma, L. Pastewka, J. Otschik, P. Gumbsch and M. Moseler, Carbon, 2015, 87, 317-329.

22 T. Ma, L. Wang, Y. Hu, X. Li and H. Wang, Sci. Rep., 2014, 4, 3662.

23 T. Kunze, M. Posselt, S. Gemming, G. Seifert, A. R. Konicek, R. W. Carpick, L. Pastewka and M. Moseler, Tribol. Lett., 2014, 53, 119-126.

24 A. Erdemir, Surf. Coat. Technol., 2001, 146-147, 292-297.

25 C. Donnet, J. Fontaine, A. Grill and T. Le Mogne, Tribol. Lett., 2000, 9, 137-142.

26 J. Martin, M. D. B. Bouchet, C. Matta, Q. Zhang, W. A. Goddard, S. Okuda and T. Sagawa, J. Phys. Chem. C, 2010, 114, 5003-5011.

27 A. R. Konicek, D. S. Grierson, A. V. Sumant, T. A. Friedmann, J. P. Sullivan, P. U. P. A. Gilbert, W. G. Sawyer and R. W. Carpick, Phys. Rev. B: Condens. Matter Mater. Phys., 2012, 85, 155448.

28 P. A. Romero, L. Pastewka, J. Von Lautz and M. Moseler, Friction, 2014, 2, 193-208.

29 A. R. Konicek, D. S. Grierson, P. U. P. A. Gilbert, W. G. Sawyer, A. V. Sumant and R. W. Carpick, Phys. Rev. Lett., 2008, 100, 235502.

30 Z. Wang, C. B. Wang, B. Zhang and J. Y. Zhang, Tribol. Lett., 2011, 41, 607-615.

31 L. Cui, Z. Lu and L. Wang, ACS Appl. Mater. Interfaces, 2013, 5, 5889-5893.

32 A. A. Al-Azizi, O. Eryilmaz, A. Erdemir and S. H. Kim, Langmuir, 2015, 31, 1711-1721.
33 X. Chen, T. Kato and M. Nosaka, ACS Appl. Mater. Interfaces, 2014, 6, 13389-13405.

34 J. Fontaine, J. L. Loubet, T. Le Mogne and A. Grill, Tribol. Lett., 2004, 17, 709-714.

35 L. Ji, H. Li, F. Zhao, W. Quan, J. Chen and A. H. Zhou, J. Phys. D: Appl. Phys., 2009, 42, 135301.

36 A. Erdemir, O. L. Eryilmaz, I. B. Nilufer and G. R. Fenske, Surf. Coat. Technol., 2000, 133-134, 448-454.

37 C. Wang, X. Ling, J. Shi and R. Xia, Surf. Interface Anal., 2014, 46, 550-555.

38 C. Wang, J. Shi, R. Xia and Z. Geng, Surf. Interface Anal., 2014, 45, 800-804.

39 G. Liu, Y. Zhou, B. Zhang, K. Gao, L. Qiang and J. Zhang, RSC Adv., 2016, 6, 59039-59044.

40 Y. Wang, K. Gao and J. Zhang, J. Appl. Phys., 2016, 120, 045303.

41 A. Erdemir, M. Switala, R. Wei and P. Wilbur, Surf. Coat. Technol., 1991, 50, 17-23.

42 M. J. Marino, E. Hsiao, L. C. Bradley, O. L. Eryilmaz, A. Erdemir and S. H. Kim, Tribol. Lett., 2011, 42, 285-291.

43 L. Wang, R. Zhang, U. Jansson and N. Nedfors, Sci. Rep., 2015, 5, 11119.

44 K. D. Koshigan, F. Mangolini, J. B. McClimon, B. Vacher, S. Bec, R. W. Carpick and J. Fontaine, Carbon, 2015, 93, 851-860.

45 C. Su and J. C. Lin, Surf. Sci., 1998, 406, 149-166.

46 J. A. Heimberg, K. J. Wahl, I. L. Singer and A. Erdemir, Appl. Phys. Lett., 2001, 78, 2449.

47 H. Li, T. Xu, C. Wang, J. Chen, H. Zhou and H. Liu, J. Phys. D: Appl. Phys., 2005, 38, 62-69.

48 H. Li, T. Xu, C. Wang, J. Chen, H. Zhou and H. Liu, Appl. Surf. Sci., 2005, 249, 257-265.

49 H. Li, T. Xu, C. Wang, J. Chen, H. Zhou and H. Liu, Diamond Relat. Mater., 2006, 15, 1228-1234.

50 H. Li, T. Xu, C. Wang, J. Chen, H. Zhou and H. Liu, Diamond Relat. Mater., 2006, 15, 1585-1592.

51 H. Li, T. Xu, C. Wang, J. Chen, H. Zhou and H. Liu, Tribol. Int., 2007, 40, 132-138.

52 H. Li, T. Xu, C. Wang, J. Chen, H. Zhou and H. Liu, Tribol. Lett., 2005, 19, 231-238.

53 C. Donnet, Surf. Coat. Technol., 1996, 80, 151-156.

54 Y. Qi, E. Konca and A. T. Alpas, Surf. Sci., 2006, 600, 29552965.

55 Y. Morita, T. Shibata, T. Onodera, R. Sahnoun, M. Koyama, H. Tsuboi, N. Hatakeyama, A. Endou, M. Kubo and C. A. D. Carpio, Jpn. J. Appl. Phys., 2008, 47, 3032-3035.

56 L. Cui, Z. Lu and L. Wang, Appl. Surf. Sci., 2015, 356, 10821085.

57 F. G. Sen, Y. Qi and A. T. Alpas, Acta Mater., 2011, 59, 2601-2614.

58 K. Hayashi, K. Tezuka, N. Ozawa, T. Shimazaki, K. Adachi and M. Kubo, J. Phys. Chem. C, 2011, 115, 22981-22986.

59 L. Wang, L. Cui, Z. Lu and H. Zhou, Carbon, 2016, 100, 556563.

60 L. Zhang, F. Wang, L. Qiang, K. Gao, B. Zhang and J. Zhang, RSC Adv., 2015, 5, 9635-9649.

61 A. Alazizi, A. Draskovics, G. Ramirez, A. Erdemir and S. H. Kim, Langmuir, 2016, 32, 1996-2004. 